\title{
Clinical Implications of the Associations Between Intestinal Microbiome and Colorectal Cancer Progression
}

This article was published in the following Dove Press journal: Cancer Management and Research

\author{
Yongkang Chen' \\ Yong Yang (D)' \\ Jin $\mathrm{Gu} \mathbb{D}^{1-3}$
}

'Department of Gastrointestinal Surgery III, Key Laboratory of Carcinogenesis and Translational Research (Ministry of Education), Peking University Cancer Hospital \& Institute, Beijing 100142, People's Republic of China; ${ }^{2}$ Department of Gastrointestinal Surgery, Peking University Shougang Hospital, Beijing 100I44, People's Republic of China; ${ }^{3}$ Peking-Tsinghua Center for Life Science, Tsinghua University, Beijing I00I42, People's Republic of China
Correspondence: Jin $\mathrm{Gu}$

Tel +86-010-88196238

Email zlguj@bjmu.edu.cn

\begin{abstract}
Intestinal microbiome influences host immunity and several diseases, including cancer, in their areas of colonization. Microbial dysbiosis and over-colonization of specific microbes within the colorectal mucosa can impact the progress of carcinogenesis. Investigations initially focused on the mechanisms by which the intestinal microbiome initiates or promotes the development of colorectal cancer, including DNA damage, induction of chromosomal instability, and regulation of host immune responses. Some studies on the clinicopathological features have reported that specific strains present at high abundance are associated with advanced stage and positive lymph nodes in colorectal cancer. In this context, we reviewed the relationship between the intestinal microbiome and the clinical features (patient age, disease staging, prognosis, etc.) of patients with colorectal cancer, and evaluated the potential pathogenesis caused by the intestinal microbiome in disease progress. This article assessed whether changes in distinct species or strains occur during the period of cancer advancement. Overall, age grouping does not bring about significant differences in the constitution of microbiome. The disease stages show their distinct distribution in some species and strains. Oncogenic species are generally enriched in patients with poor prognosis, including low infiltration of $\mathrm{CD}^{+} \mathrm{T}$ cells, poor differentiation, widespread invasion, high microsatellite instability, $\mathrm{CpG}$ island methylator phenotype, BRAF mutation, short overall survival, and disease-free survival. The implications of those changes we discussed may assist in comprehensive understanding of the tumorigenesis of colorectal cancer from a microbiological perspective, finding potential biomarkers for colorectal cancer.
\end{abstract}

Keywords: colorectal cancer, intestinal microbiome, patient age, disease stage, prognosis

\section{Introduction}

Recent research on human symbiotic microbiome has revealed its significant impact on human health. Associations have been shown between microbiota and diabetes, liver diseases, hypertension, and chronic kidney diseases ${ }^{1-3}$. The microbiota also plays a significant role in cancer development through inflammation, DNA damage, and cellular immunity ${ }^{4-9}$. Since the colorectum is the area most densely populated by the microbiota in vivo, the roles of the intestinal microbiome in the processes of colorectal cancer development are of scientific interest. ${ }^{10}$

The latest Global Cancer Incidence, Mortality, and Prevalence (GLOBOCAN) statistics indicate that colorectal cancer is the second leading cause of cancerassociated deaths worldwide. ${ }^{11}$ Lifestyle and diet are closely related to sporadic colorectal cancer. ${ }^{12}$ Improper diets alter the composition, abundance, and balance of 
the intestinal microbiome, destabilizing the stasis between microbiota and the intestinal epithelium, resulting in intestinal inflammation and cancer. ${ }^{13}$ Even familial colorectal polyposis with a genetic predisposition may develop early carcinogenesis due to epithelial damage caused by interactions between specific intestinal bacterial types and interleukin-17. ${ }^{14}$ Some investigators have questioned the vital role of the microbiome, reporting intestinal microbial changes are accompanied consequences in tumor formation. ${ }^{15}$ However, mice have been shown to be more susceptible to colorectal dysplasia after being fed feces from patients with colorectal cancer. ${ }^{16}$

The intestinal microbiome can not only initiate the tumorigenesis of colonic epithelial cells but promote the growth and metastasis of cancers that have already developed. Specific colonizing bacteria identified in the cancerous colorectal mucosa have been shown to be diverse from those found in mucosa adjacent to the tumor, in patients with benign colorectal diseases, and in healthy individuals. ${ }^{17,18}$ Moreover, recent reports addressed that colonization of specific species was associated with positive regional lymph nodes, vessel carcinoma embolus, and gene mutations. ${ }^{19,20}$ These relations give those distinctive gut bacteria and their metabolites the potential to become non-invasive biomarkers of colorectal cancer. Recently, this review focused on recent changes in the composition, abundance, and balance of intestinal microbiome in colorectal cancer and their relationship to the clinical feature (age, stage, and prognosis) of patients (Table 1), to explore the evidence of their influence on the development of colorectal cancer.

\section{Literature Search}

\section{Characteristics of Intestinal Microbiota in Colorectal Cancer}

Analysis of the 16S rRNA gene sequence in feces or colorectal tissues has identified distinctive changes in intestinal microbiota. The overall microbiota diversity (i.e., the number of different taxa) declined in patients with colorectal cancer; however, the microbial diversity in those patients was not significantly different compared to that in the control group (i.e., the distribution of taxa). ${ }^{21}$ Comparison of taxonomic groups based on phyla between cancer patients and control volunteers revealed significant enrichment of Fusobacteria (to the greatest extent), Proteobacteria, and Bacteroidetes in CRC patients. In contrast, Actinobacteria and Firmicutes were relatively depleted. ${ }^{18,22}$ Another study reported the enrichment of Fusobacterium, Porphyromonas, and Atopobium in colorectal cancer. ${ }^{21}$ Firmicutes depletion was commonly observed in several studies. Clostridia was the most significantly reduced among Firmicutes compared to the declines in Coprococcus and Lachnospiraceae. ${ }^{18,21}$ Coprococcus ferments dietary fiber and other complex carbohydrates into butyric acids, the primary metabolite that inhibits colonic inflammation and tumorigenesis. ${ }^{21} \mathrm{~A}$ Chinese study reported a relative reduction in butyrate-producing bacteria (Clostridiaceae, Ruminococcus, etc.), suggesting that these bacteria could protect the colorectal mucosa from carcinogenesis. ${ }^{23}$

Some studies have focused on distinctive microbiota in colorectal cancer according to species taxonomy. A metagenomic classifier for colorectal cancer detection revealed that the four most discriminative species included two Fusobacterium species, Porphyromonas asaccharolytica and Peptostreptococcus stomatis, and two subspecies, F. nucleatum vincentii and $F$. nucleatum animalis, which distinguished colorectal cancer with an accuracy of $0.63 .^{22}$ A study that characterized bacterial communities in stool samples found that colorectal cancer-associated bacteria belonged to taxa commonly associated with periodontal disease, ${ }^{22,24}$ including Pophyromonas asaccharolytica, Fusobacterium nucleatum, Parvimonas micra, Peptostreptococcus stomatis, Gemella spp., and an unclassified Prevotella. ${ }^{22}$

Integrated microbial genome (IMG of species), operational taxonomic unit (OTU), and metagenomic linkage group (MLG) analysis revealed three oral pathogens, Parvimonas micra, Fusobacterium nucleatum, and Solobacterium moorei to be enriched in colorectal cancer tissue, while over-colonization of Peptostreptococcus stomatis was only detected by two of these methods. ${ }^{25} \mathrm{~A}$ multicenter metagenome sequencing study among China, Austria, the United States, Germany, and France confirmed that seven distinctive bacteria are over-represented in colorectal cancer (Bacteroides fragilis, Fusobacterium nucleatum, Porphyromonas asaccharolytica, Parvimonas micra, Prevotella intermedia, Alistipes finegoldii, and Thermanaerovibrio acidaminovorans). ${ }^{26}$ Besides, levels of Peptostreptococcus anaerobius were elevated in colorectal cancer mucosa compared to those in colorectal polyps. ${ }^{27}$ Discrepancy analysis revealed significant enrichment of Enterococcus faecalis, Enterotoxigenic Bacteroides fragilis (ETBF) in carcinoma tissues compared to that in tumor-adjacent tissues in colorectal cancer. ${ }^{28}$ In contrast, levels of Eubacterium rectale and Faecalibacterium prausnitzii (both butyric acid-producing bacteria) were reduced by four-fold in colorectal 


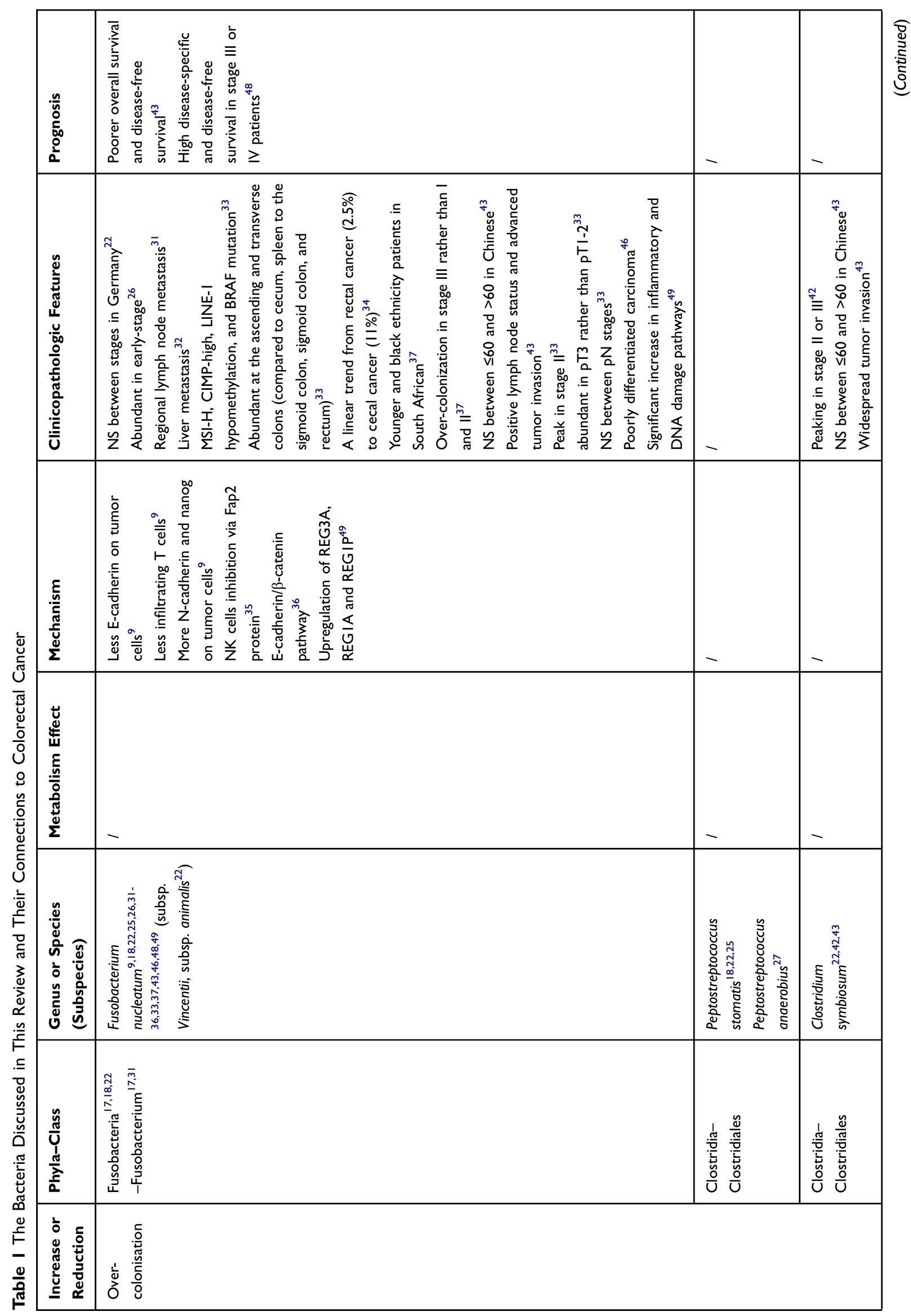




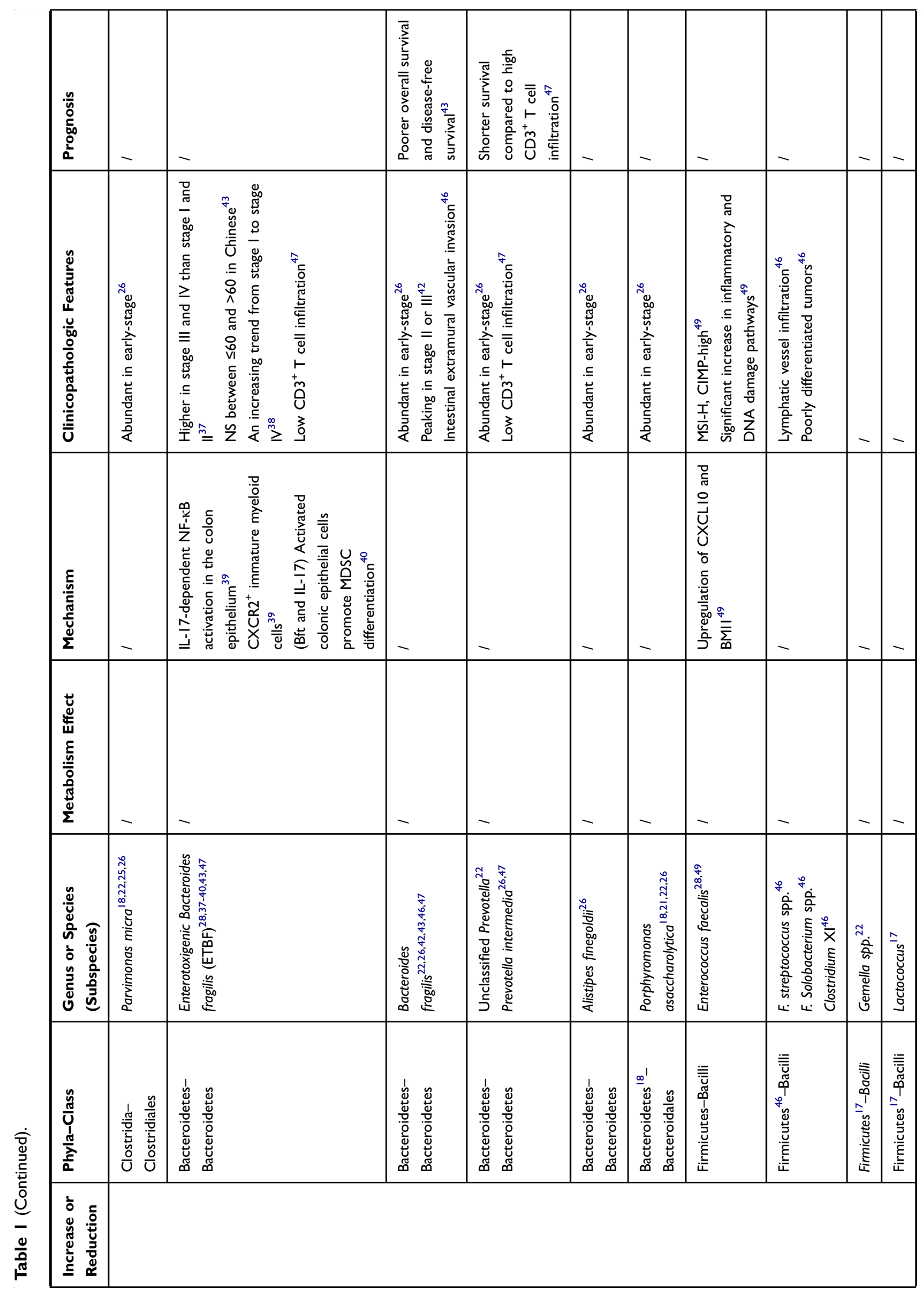




\begin{tabular}{|c|c|c|c|c|c|c|c|c|c|c|c|c|}
\hline- & - & - & - & 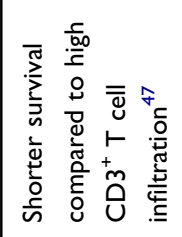 & - & - & - & - & - & - & - & - \\
\hline 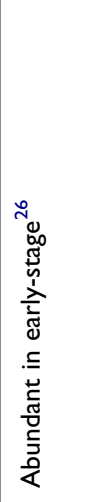 & 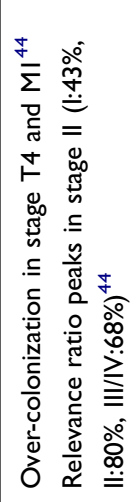 & 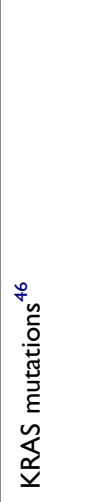 & - & 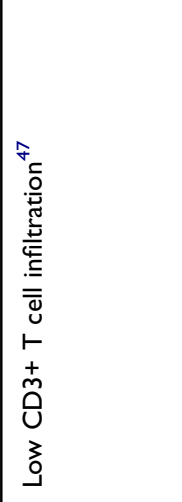 & - & - & - & - & 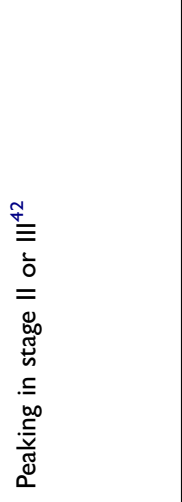 & - & - & 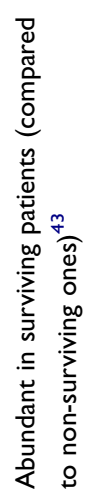 \\
\hline- & 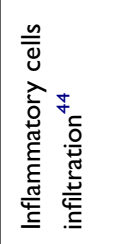 & - & - & - & - & - & - & - & - & - & - & - \\
\hline - & - & - & - & - & - & - & - & - & 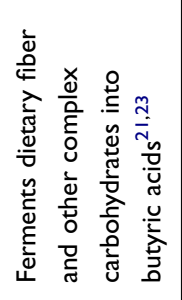 & 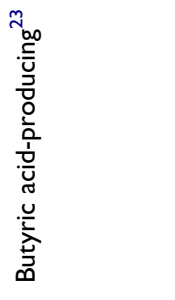 & 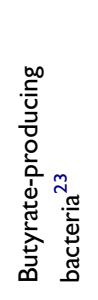 & - \\
\hline 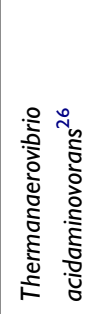 & 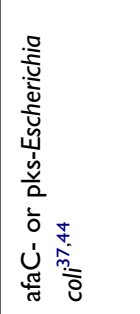 & 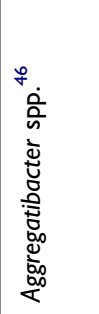 & 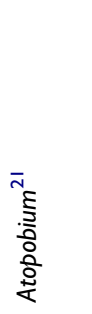 & 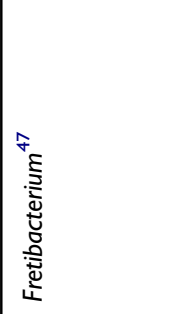 & 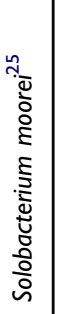 & 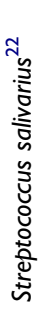 & 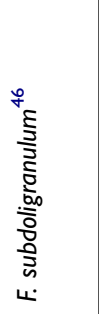 & 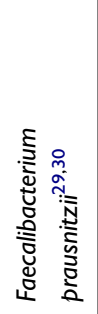 & 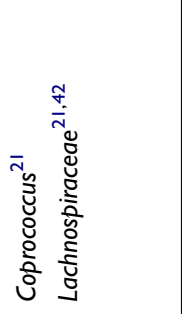 & 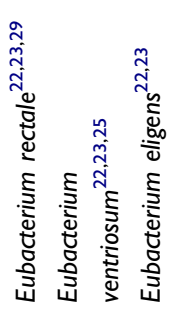 & 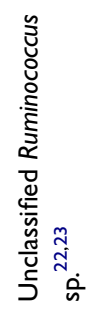 & 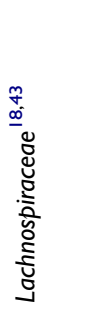 \\
\hline 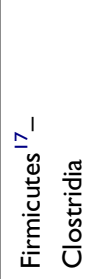 & 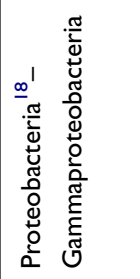 & 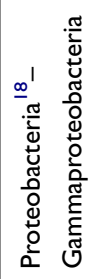 & 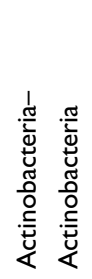 & - & - & 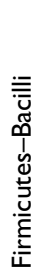 & 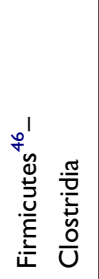 & 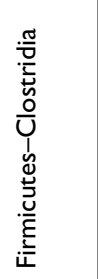 & 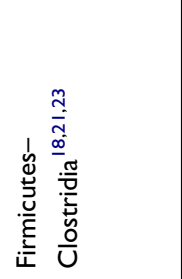 & 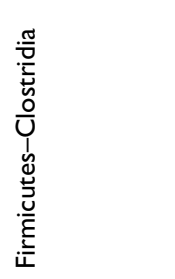 & 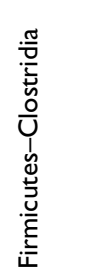 & 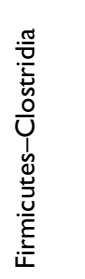 \\
\hline & & & & & & \multicolumn{7}{|c|}{ 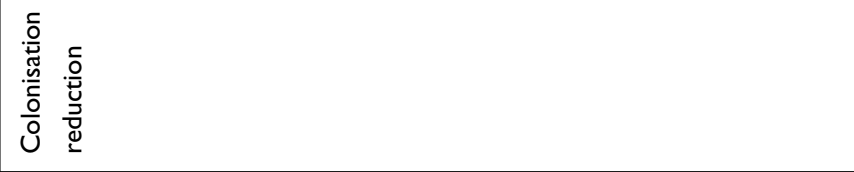 } \\
\hline
\end{tabular}




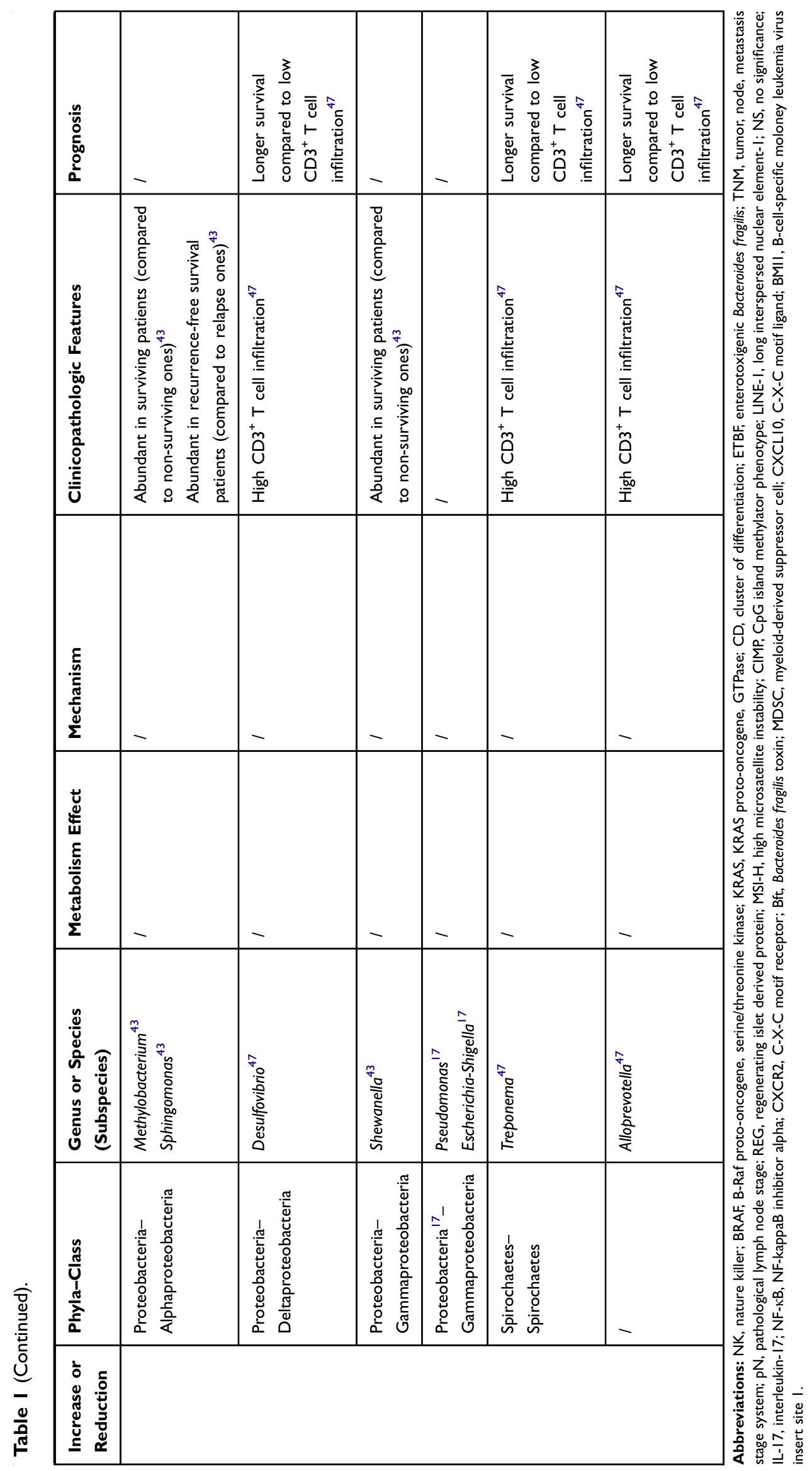


cancer patients compared to those in healthy control volunteers. $^{29,30}$ Also, Eubacterium ventriosum showed higher levels in control microbiomes compared to those in colonic cancerous microbiomes. ${ }^{25}$

Studies on Fusobacterium and the development of colorectal cancer are more abundant and thorough than those on any other cancer-related microbiome. DNA extracted from tissues in a cohort study revealed that the average total abundance of Fusobacterium in tumor samples was 415 times higher than those of matched healthy samples. ${ }^{31}$ Data from The Cancer Genome Atlas (TCGA) cohort showed that microorganisms present at the metastatic niche in the liver of patients with Fusobacterium-positive colorectal cancer were similar to those in the primary site, suggesting that the high relative abundance of Fusobacterium significantly increases the risk of liver metastasis. ${ }^{32}$ To exclude the potential confounding of the results due to a connection between Fusobacterium and liver tissues, Fusobacterium sequencing of primary hepatic carcinoma and metastatic tumor revealed deficient concentrations of Fusobacterium in primary liver cancer. ${ }^{32}$ Fusobacterium nucleatum appears to play a significant role in the Fusobacterium over-colonization of colorectal cancer mucosa. ${ }^{31}$ Rather than the other colorectal sites (cecum, spleen to the sigmoid colon, sigmoid colon, and rectum), Fusobacterium nucleatum appears to prefer the ascending and transverse colons. ${ }^{33}$ The proportions of cancer patients with high relative abundances of Fusobacterium nucleatum increased from $2.5 \%(4 / 157)$ for rectal cancer to $11 \%(19 / 178)$ for cecal cancer, a linear and significant trend. ${ }^{34}$ Compared with other sites, a reduction in the abundance of Fusobacterium nucleatum in the rectum, ascending colon, and cecum is often observed. ${ }^{34}$ Consistent with the conclusions above, Fusobacterium nucleatum present in both the primary site and the corresponding metastatic tumor is more likely to be abundant in metastatic carcinomas of the cecum and ascending colon rather than in other sites. ${ }^{32}$ Fusobacterium nucleatum can inhibit positive immune from natural killer cells by Fap2 protein, inducing immune evasion. ${ }^{35}$ Moreover, Fusobacterium nucleatum can utilize the E-cadherin/ $\beta$-catenin pathway to not only increase susceptibility to colorectal tumorigenesis but also exacerbate cancer cell proliferation and invasion. ${ }^{36}$

In addition to Fusobacterium nucleatum, other strains with distinctive pathogenic factors have also attracted attention. The presence of enterotoxigenic Bacteroides fragilis (ETBF) and afaC or polyketide synthase (pks)-positive Escherichia coli (afaC- or pks- E. coli) was significantly associated with colorectal cancer. ${ }^{37}$ Bacteroides fragilis toxin (bft) is a toxic protein secreted by ETBF. Among diverse bft isoforms, bft-1, and bft- 2 were more commonly detected in colorectal cancer cases $(67.8 \%)$ than in the control group (34.4\%). ${ }^{38}$ A study utilizing transgenic mice found that CXCR2-positive multinuclear immature myeloid cells, which are recruited by the CXCL1 concentration gradient, cooperate with ETBF to initiate distal colonic tumorigenesis with IL-17-dependent NF- $\kappa$ B activation in the epithelium of colorectal cancer tissue. ${ }^{39}$ Colonic epithelial cells activated by Bft and IL-17 promote differentiation from macrophages to marrow-derived suppressor cells, which selectively upregulate arginase 1 , nitric oxide synthase 2 , and nitric oxide, finally inhibiting $\mathrm{T}$ cell growth. ${ }^{40} \mathrm{Pks}$-positive E.coli can affect the progression of colorectal cancer by aggravating the infiltration of inflammatory cells. ${ }^{41}$

\section{Intestinal Microbes in Colorectal Cancer Patients of Different Ages}

Although a few recent studies have focused on the differences in intestinal microbiota between different age groups of patients with colorectal cancer, there are many clues. A similar enrichment was widely found between elderly (over 65 years of age) and younger (not over 65 years) patients in macrogene linkage group analysis, indicating that cancerassociated microbiota in diverse age groups shares common features. $^{42}$ An investigation of the prognosis of colorectal cancer patients with over-representation of Fusobacterium nucleatum, Bacteroides fragilis, or Clostridium, observed no significant differences in the relative abundances of the $16 \mathrm{~S}$ RNA levels of these bacteria in mucosal tissues between older (over 60 years) and younger patients (less than 60 years). ${ }^{43}$ Fisher's exact tests of the correlation between high-abundance Fusobacterium and clinical factors revealed no association between Fusobacterium and patient age. ${ }^{31}$ However, a South African study reported that a high level of Fusobacterium colonization of colorectal cancer mucosa was more likely to be present in younger patients and those of black ethnicity. ${ }^{37}$ Patients with low- and moderate-level colonization were predominantly between 60-70 years of age, while high-level colonization occurred mostly in patients 50-60 years of age, a statistically significant difference. About $31 \%$ of patients below 60 years of age, and $11 \%$ of those above 60 years had high levels of Fusobacterium colonization. ${ }^{37}$ Differences in these findings between studies may be related to differences in ethnicity. Collectively, it appears that significant age-associated changes in the intestinal microbiome have not been observed. 


\section{Intestinal Microbes in Different Stages of Colorectal Cancer}

Evidence suggests the presence of distinctive enrichment patterns of intestinal microbes according to the disease stage. A metagenomic sequencing study observed that seven of the 126 macrogene linkage groups (MLG-190, MLG-603, MLG-604, MLG-629, MLG-219, MLG-893, and MLG-1002; each MLG contained >100 genes) extracted from intestinal mucosa differed significantly between stages, peaking in patients with stage II or III colorectal cancer. These seven MLGs included gene groups shared by Lachnospiraceae bacterium, Clostridium symbiosum, and Bacteroides ssp. ${ }^{42}$ In the multi-national metagenomic sequencing project previously mentioned, Bacteroides fragilis, Fusobacterium nucleatum, Porphyromonas asaccharolytica, Parvimonas micra, Prevotella intermedia, Alistipes finegoldii, and Thermanaerovibrio acidaminovorans, which were distinctively enriched in 526 intestinal mucosa colorectal cancer samples, were more likely to be abundant in early-stage than late-stage disease. ${ }^{26}$ In contrast, a metagenomic classifier study in Germany using species and subspecies of Fusobacterium detected microbe changes in both early-stage (I and II) and late-stage (III and IV) disease. ${ }^{22}$

Furthermore, Fusobacterium are more likely to exhibit over-colonization in stage III colonic cancerous mucosa than in stage I or stage II disease. ${ }^{37}$ Patients with pathological T3 stage (pT3, subserosal layer) disease had more copies of Fusobacterium nucleatum DNA in their tumor tissues compared to that in patients with stage T1 or T2 disease; however, a similar phenomenon was not observed for the pathological N stage. ${ }^{33}$ The enrichment of Fusobacterium nucleatum peaks in AJCC stage II, which is correlated to highly differentiated colonic carcinoma. ${ }^{33}$

Levels of ETBF are higher in stage III and IV disease than those in stage I and stage II colorectal cancer. A similar finding was also reported in a comparison between stage I and IV tumor-adjacent mucosa. ${ }^{37}$ Moreover, the enterotoxinencoding gene from Bacteroides fragilis was widely detected in the intestinal mucosa of patients with colorectal cancer, especially in those with stage III or IV disease, indicating that the bft gene may be a risk factor for advanced colorectal cancer. The rate of mucosal bft positivity in colorectal cancer showed an increasing trend between stage I and stage IV, although this trend was not statistically significant $(72.7 \%$ and $100 \%$, respectively). ${ }^{38}$ In order to eliminate confounding in the microbiome detection resulting from preoperative antibiotics, bft detection was done among patients with different stages of colorectal cancer undergoing mechanical preparations and not oral administration of antibiotics, with similar frequencies to those observed previously. ${ }^{38}$

E. coli strain $11 \mathrm{G} 5$, a member of the $\mathrm{B} 2$ phylogroup, possesses a pks gene island encoding colibactin. It is considered the representative colon cancer-associated E. coli strain because of its high levels of colonization of the tumor-adjacent mucosa and cancerous tissues of patients with advanced cancer (T4) and liver metastasis (M1). ${ }^{44}$ Compared to stage I (43\%), B2 phylogroup E. coli is more likely to be detected in patients with stage II $(80 \%)$ and stage III/IV (68\%) disease. Cyclomodulin-positive E. coli strains throughout the entire colorectal mucosa or tumor tissues are found significantly more often in patients with TNM stage II $(64 \%)$ and III/IV (67\%) disease than in those with stage I disease $(45 \%)$. The colonization level of E. coli in the mucosa or tumor had no impact on the ratio of these two $E$. coli strains. ${ }^{44}$ The detection frequency in patients with stage II-IV colorectal cancer (59.3\%) was significantly increased compared to that in patients with stage I disease $(0.3 \%){ }^{45}$

\section{Intestinal Microbes and Prognosis in Patients with Colorectal Cancer}

A growing body of evidence supports that intestinal microbes provide clues for colorectal cancer with poor prognosis. Analysis of the Chao diversity index showed a lower microbial community diversity in recurrence-free survival patients than that of patients with recurrent colorectal cancer. ${ }^{21}$ Analysis of the relative abundance of OTU demonstrated diverse compositions in the studied groups in patients with various prognoses (death, survival with relapse, and survival without relapse). Proteobacteria (33.8-49.4\%), Firmicutes (16.9-22.7\%), Bacteroidetes (21.1-27.9\%), and Fusobacterium (3.38-10.8\%) predominated in these groups. ${ }^{43}$ High-abundance Bacteroides is associated with intestinal extramural vascular invasion of colorectal cancer. At the same time, Firmicuteria is more likely to be connected to the presence of lymphatic vessel infiltration and Proteobacteria, Aggregatibacter spp. are related to KRAS mutations. ${ }^{46}$ Wei et al reported a relatively higher abundance of Shewanella, Methylobacterium, Faecalibacterium, and Sphingomonas in surviving patients than that in non-surviving patients. Methylbacteria had a higher relative abundance in recurrence-free survival patients compared to that in patients with relapse. ${ }^{43}$ Specific bacteria, including Alloprevotella, Treponema, and Desulfovibrio are significantly enriched in colorectal 
cancer with high $\mathrm{CD}^{+} \mathrm{T}$ cell infiltration, whereas Prevotella, Bacteroides, and Fretibacterium are widely detected in cancers with low infiltration of $\mathrm{CD}^{+} \mathrm{T}$ cells. Furthermore, patients with high CD3 density had a significantly longer survival compared to that in patients with low CD3 density. ${ }^{47}$

At the species level, $F$. streptococcus spp., $F$. Solobacterium spp., and Clostridium XI spp., which belong to Firmicuteria, were more likely to exhibit over-colonization in poorly differentiated tumors, while $F$. subdoligranulum was detected at a low frequency. ${ }^{46}$ In addition, a relative abundance above $0.52 \%$ in the colonic mucosa was defined as high-abundance Fusobacterium nucleatum, which was significantly associated with positive lymph node status and advanced tumor invasion. An abundance of $0.55 \%$ for Clostridium was only significantly associated with widespread tumor invasion. $^{43}$

The presence of Fusobacterium, especially Fusobacterium nucleatum, is highly correlated with severe invasion and poor prognosis of colorectal cancer. A higher relative abundance of Fusobacterium likely exists in poorly differentiated carcinoma. $^{46}$ Moreover, the relative abundance of Fusobacterium nucleatum is negatively correlated with E-cadherin on the surface of tumor cells and cancerous tissue-infiltrating T cells. ${ }^{9}$ In contrast, its relative abundance is positively correlated with N-cadherin and Nanog (a gene thought to maintain self-renewal of embryonic stem cells), suggesting the epithelial-mesenchymal transition of colorectal cancer cells. ${ }^{48}$ Tumors with relatively abundant Fusobacterium are more likely to have regional lymph node metastasis, as indicated by the TNM score. ${ }^{31}$ Specifically, the proportions of patients with lymph node metastasis with high and low abundances of Fusobacterium were $74 \%$ and $45 \%$, respectively. ${ }^{31}$ The colorectal cancer-specific mortality among patients with low (multivariate hazard ratio [HR]: 1.25; 95\% confidence interval [CI]: 0.82-1.92) or high (HR:1.58, 95\% CI: 1.04-2.39) loads of Fusobacterium nucleatum in their colonic mucosa was significantly higher than that in Fusobacterium nucleatum-negative patients. $^{33}$ A high Fusobacterium nucleatum load was associated with high microsatellite instability (MSI) (odds ratio: 5.22, 95\% CI: 2.86-9.55) independent of the $\mathrm{CpG}$ island methylator phenotype (CIMP) and BRAF mutation status in multivariable logistic regression analysis. In this study, Fusobacterium nucleatum was also associated with CIMP-high, LINE-1 hypomethylation, and BRAF mutation. ${ }^{33}$ Another study reported by Lennard showed that activation in inflammatory and DNA damage pathways was strong relative to Fusobacterium nucleatum along with Enterococcus faecalis. ${ }^{49}$ Colorectal cancer with dMMR/MSI has specific phenotypes, including proximal colon preference, poor differentiation, and lymphocyte enrichment $^{50-52}$. This study revealed that these two spaces might boost the disease development and progression via a transcriptional remodeling, including an increase of regenerating islet derived protein (REG3A, REG1A, and REG1P, highabundance Fusobacterium nucleatum) and up-regulation of CXCL10 and BMI1 (high-abundance E. faecalis). ${ }^{49}$ These findings indicate the association between the Fusobacterium nucleatum load in colorectal cancer tissue and poor survival. No or low fold-increase of Fusobacterium nucleatum in tumor tissues relative to the matched tumor-adjacent tissues in colorectal cancer resulted in a significant advantage in survival over patients with high fold-increases (no fold increase: $<25$, high fold-increase: $>216$ ). The median survival in patients with high- and low-fold Fusobacterium nucleatum was two and three years, respectively. ${ }^{53}$ Both disease-specific and diseasefree survival were higher in patients with stage III or IV colorectal cancer with a lower relative abundance of Fusobacterium nucleatum compared to survival in patients with higher relative DNA expression. ${ }^{48}$

Wei et al reported a significantly more reduced three-year overall survival in patients with high abundances of Bacteroides fragilis and Fusobacterium nucleatum compared to that in patients with low abundances of these strains based on Kaplan-Meier analysis and Log rank tests of the relationship between patient survival and relative bacterial abundance in colorectal cancer tissues. ${ }^{43}$ Besides, patients with a low abundance of Clostridium had a poor three-year overall survival, but the difference was not statistically significant. ${ }^{43}$ Similarly, patients with high abundances of Bacteroides fragilis and Fusobacterium nucleatum had lower disease-free survival rates than those in low-abundance patients. ${ }^{43}$ Cox regression and multivariate analysis showed that over-representation of Bacteroides fragilis (HR:2.010; 95\% CI:1.0203.961) and Fusobacterium nucleatum (HR:1.993; 95\% CI:1.024-3.879) was associated with low overall survival after radical surgery in patients with colorectal cancer, which were independent predictors for three-year overall survival. $^{43}$

\section{Conclusion}

The intestinal microbiome involves in the development of colorectal cancer by altering the intestinal epithelial cells and the balance of the immune microenvironment. The species present in Fusobacteria, Proteobacteria, Firmicutes, Bacteroidetes, and Clostridia mainly play an indispensable 
role in promoting the growth of tumors already present. The interaction between gut microbiota and colorectal cancer is multi-level and manifold. Different species in the microflora have a different impact on the malignant behaviors of the tumor. Molecular mutations (KRAS and BRAF), regional lymph node metastasis, and $\mathrm{CD}^{+} \mathrm{T}$ cells inhibition are some of their potential targets. Their associations make assessing changes in the intestinal microbiome a convenient predictive tool for colorectal cancer. Useful biomarkers can influence treatment strategies for patients with colorectal cancer. Specific microbial can mediate the response to chemotherapy and radiotherapy, resulting in changing the prognosis of patients. Analysis of the gut microbiome offers the potential to develop non-invasive diagnostic tests that can serve as filtrating markers for improving treatment response. The potential of regulating the intestinal microbiome via changing the diet and using probiotics provides hope for reducing the risk of cancer development and improving the effectiveness of treatment.

\section{Data Sharing Statement}

The datasets analyzed during the current study are available from the corresponding author on reasonable request.

\section{Acknowledgment}

Thanks to Jiajia Chen for sharing the Bacteria Classification Table.

\section{Funding}

This work was funded by Special Projects of Prevention and Control of Non-Communicable Diseases (2016YFC1302605) and Beijing Municipal Science \& Technology Commission, Clinical Application and Development of Capital Characteristic (Z171100001017087).

\section{Disclosure}

All authors declare no conflicts of interest.

\section{References}

1. Li Q, Gao Z, Wang H, et al. Intestinal immunomodulatory cells ( $\mathrm{T}$ Lymphocytes): a bridge between gut microbiota and diabetes. Mediators Inflamm. 2018;2018:9830939. doi:10.1155/2018/9830939

2. Altamirano-Barrera A, Uribe M, Chavez-Tapia NC, Nuno-Lambarri N. The role of the gut microbiota in the pathology and prevention of liver disease. J Nutr Biochem. 2018;60:1-8. doi:10.1016/j.jnutbio.2018. 03.006

3. Yang T, Richards EM, Pepine CJ, Raizada MK. The gut microbiota and the brain-gut-kidney axis in hypertension and chronic kidney disease. Nat Rev Nephrol. 2018;14(7):442-456. doi:10.1038/s41581018-0018-2
4. Schwabe RF, Jobin C. The microbiome and cancer. Nat Rev Cancer. 2013;13(11):800-812. doi:10.1038/nrc3610

5. Dapito DH, Mencin A, Gwak GY, et al. Promotion of hepatocellular carcinoma by the intestinal microbiota and TLR4. Cancer Cell. 2012;21(4):504-516. doi:10.1016/j.ccr.2012.02.007

6. Drewes JL, Housseau F, Sears CL. Sporadic colorectal cancer: microbial contributors to disease prevention, development and therapy. $\mathrm{Br}$ $J$ Cancer. 2016;115(3):273-280. doi:10.1038/bjc.2016.189

7. Viaud S, Saccheri F, Mignot G, et al. The intestinal microbiota modulates the anticancer immune effects of cyclophosphamide. Science. 2013;342(6161):971-976. doi:10.1126/science.1240537

8. Iida N, Dzutsev A, Stewart CA, et al. Commensal bacteria control cancer response to therapy by modulating the tumor microenvironment. Science. 2013;342(6161):967-970. doi:10.1126/science. 1240527

9. Vetizou M, Pitt JM, Daillere R, et al. Anticancer immunotherapy by CTLA-4 blockade relies on the gut microbiota. Science. 2015;350 (6264):1079-1084. doi:10.1126/science.aad1329

10. Nakatsu G, Li X, Zhou H, et al. Gut mucosal microbiome across stages of colorectal carcinogenesis. Nat Commun. 2015;6:8727. doi: $10.1038 /$ ncomms 9727

11. Bray F, Ferlay J, Soerjomataram I, Siegel RL, Torre LA, Jemal A. Global cancer statistics 2018: GLOBOCAN estimates of incidence and mortality worldwide for 36 cancers in 185 countries. CA Cancer J Clin. 2018;68(6):394-424. doi:10.3322/caac.21492

12. Wiseman M. The Second World Cancer Research Fund/American Institute for Cancer Research Expert Report. food, nutrition, physical activity, and the prevention of cancer: a global perspective. Proc Nutr Soc. 2008;67(3):253-256. doi:10.1017/S002966510800712X

13. Han S, Gao J, Zhou Q, Liu S, Wen C, Yang X. Role of intestinal flora in colorectal cancer from the metabolite perspective: a systematic review. Cancer Manag Res. 2018;10:199-206. doi:10.2147/CMAR.S153482

14. Dejea CM, Fathi P, Craig JM, et al. Patients with familial adenomatous polyposis harbor colonic biofilms containing tumorigenic bacteria. Science. 2018;359(6375):592-597. doi:10.1126/science.aah3648

15. Tjalsma H, Boleij A, Marchesi JR, Dutilh BE. A bacterial driverpassenger model for colorectal cancer: beyond the usual suspects. Nat Rev Microbiol. 2012;10(8):575-582. doi:10.1038/nrmicro2819

16. Wong SH, Zhao L, Zhang X, et al. Gavage of fecal samples from patients with colorectal cancer promotes intestinal carcinogenesis in germ-free and conventional mice. Gastroenterology. 2017;153 (6):1621-1633 e1626. doi:10.1053/j.gastro.2017.08.022

17. Gao Z, Guo B, Gao R, Zhu Q, Qin H. Microbiota disbiosis is associated with colorectal cancer. Front Microbiol. 2015;6:20. doi:10.3389/fmicb.2015.00020

18. Baxter NT, Ruffin M, Rogers MA, Schloss PD. Microbiota-based model improves the sensitivity of fecal immunochemical test for detecting colonic lesions. Genome Med. 2016;8(1):37. doi:10.1186/ s13073-016-0290-3

19. Eklof V, Lofgren-Burstrom A, Zingmark C, et al. Cancer-associated fecal microbial markers in colorectal cancer detection. Int $J$ Cancer. 2017;141(12):2528-2536. doi:10.1002/ijc.31011

20. Liang Q, Chiu J, Chen Y, et al. Fecal bacteria act as novel biomarkers for noninvasive diagnosis of colorectal cancer. Clin Cancer Res. 2017;23(8):2061-2070. doi:10.1158/1078-0432.CCR-16-1599

21. Ahn J, Sinha R, Pei Z, et al. Human gut microbiome and risk for colorectal cancer. J Natl Cancer Inst. 2013;105(24):1907-1911. doi:10.1093/jnci/djt300

22. Zeller G, Tap J, Voigt AY, et al. Potential of fecal microbiota for early-stage detection of colorectal cancer. Mol Syst Biol. 2014;10:766. doi:10.15252/msb.20145645

23. Wang X, Ye T, Chen WJ, et al. Structural shift of gut microbiota during chemo-preventive effects of epigallocatechin gallate on colorectal carcinogenesis in mice. World J Gastroenterol. 2017;23 (46):8128-8139. doi:10.3748/wjg.v23.i46.8128 
24. Warren RL, Freeman DJ, Pleasance S, et al. Co-occurrence of anaerobic bacteria in colorectal carcinomas. Microbiome. 2013;1(1):16. doi:10.1186/2049-2618-1-16

25. Yu J, Feng Q, Wong SH, et al. Metagenomic analysis of faecal microbiome as a tool towards targeted non-invasive biomarkers for colorectal cancer. Gut. 2017;66(1):70-78. doi:10.1136/gutjnl-2015-309800

26. Dai Z, Coker OO, Nakatsu G, et al. Multi-cohort analysis of colorectal cancer metagenome identified altered bacteria across populations and universal bacterial markers. Microbiome. 2018;6(1):70. doi:10.1186/s40168-018-0451-2

27. Tsoi H, Chu ESH, Zhang X, et al. Peptostreptococcus anaerobius induces intracellular cholesterol biosynthesis in colon cells to induce proliferation and causes dysplasia in mice. Gastroenterology. 2017;152(6):1419-1433 e1415. doi:10.1053/j.gastro.2017.01.009

28. Zhou Y, He H, Xu H, et al. Association of oncogenic bacteria with colorectal cancer in South China. Oncotarget. 2016;7(49):8079480802. doi:10.18632/oncotarget.13094

29. Konstantinov SR, Kuipers EJ, Peppelenbosch MP. Functional genomic analyses of the gut microbiota for CRC screening. Nat Rev Gastroenterol Hepatol. 2013;10(12):741-745. doi:10.1038/ nrgastro.2013.178

30. Balamurugan R, Rajendiran E, George S, Samuel GV, Ramakrishna BS. Real-time polymerase chain reaction quantification of specific butyrate-producing bacteria, Desulfovibrio and Enterococcus faecalis in the feces of patients with colorectal cancer. $J$ Gastroenterol Hepatol. 2008;23(8 Pt 1):1298-1303. doi:10.1111/j.14401746.2008.05490.x

31. Castellarin M, Warren RL, Freeman JD, et al. Fusobacterium nucleatum infection is prevalent in human colorectal carcinoma. Genome Res. 2012;22(2):299-306. doi:10.1101/gr.126516.111

32. Bullman S, Pedamallu CS, Sicinska E, et al. Analysis of Fusobacterium persistence and antibiotic response in colorectal cancer. Science. 2017;358(6369):1443-1448. doi:10.1126/science.aal5240

33. Mima K, Nishihara R, Qian ZR, et al. Fusobacterium nucleatum in colorectal carcinoma tissue and patient prognosis. Gut. 2016;65 (12):1973-1980. doi:10.1136/gutjnl-2015-310101

34. Mima K, Cao Y, Chan AT, et al. Fusobacterium nucleatum in colorectal carcinoma tissue according to tumor location. Clin Transl Gastroenterol. 2016;7(11):e200. doi:10.1038/ctg.2016.53

35. Gur C, Ibrahim Y, Isaacson B, et al. Binding of the Fap2 protein of Fusobacterium nucleatum to human inhibitory receptor TIGIT protects tumors from immune cell attack. Immunity. 2015;42(2):344355. doi:10.1016/j.immuni.2015.01.010

36. Yang Y, Weng W, Peng J, et al. Fusobacterium nucleatum increases proliferation of colorectal cancer cells and tumor development in mice by activating toll-like receptor 4 signaling to nuclear factor-kappaB, and up-regulating expression of MicroRNA-21. Gastroenterology. 2017;152 (4):851-866 e824. doi:10.1053/j.gastro.2016.11.018

37. Viljoen KS, Dakshinamurthy A, Goldberg P, Blackburn JM, McDowell A. Quantitative profiling of colorectal cancer-associated bacteria reveals associations between fusobacterium spp., enterotoxigenic Bacteroides fragilis (ETBF) and clinicopathological features of colorectal cancer. PLoS One. 2015;10(3):e0119462. doi:10.1371/journal.pone. 0119462

38. Boleij A, Hechenbleikner EM, Goodwin AC, et al. The Bacteroides fragilis toxin gene is prevalent in the colon mucosa of colorectal cancer patients. Clin Infect Dis. 2015;60(2):208-215. doi:10.1093/ $\mathrm{cid} / \mathrm{ciu} 787$
39. Chung L, Thiele Orberg E, Geis AL, et al. Bacteroides fragilis toxin coordinates a pro-carcinogenic inflammatory cascade via targeting of colonic epithelial cells. Cell Host Microbe. 2018;23(2):203-214 e205. doi:10.1016/j.chom.2018.01.007

40. Thiele Orberg E, Fan H, Tam AJ, et al. The myeloid immune signature of enterotoxigenic Bacteroides fragilis-induced murine colon tumorigenesis. Mucosal Immunol. 2017;10(2):421-433. doi:10.1038/mi.2016.53

41. Veziant J, Gagniere J, Jouberton E, et al. Association of colorectal cancer with pathogenic Escherichia coli: focus on mechanisms using optical imaging. World J Clin Oncol. 2016;7(3):293-301. doi:10.5306/wjco.v7.i3.293

42. Feng Q, Liang S, Jia H, et al. Gut microbiome development along the colorectal adenoma-carcinoma sequence. Nat Commun. 2015;6:6528. doi:10.1038/ncomms 7528

43. Wei Z, Cao S, Liu S, et al. Could gut microbiota serve as prognostic biomarker associated with colorectal cancer patients' survival? A pilot study on relevant mechanism. Oncotarget. 2016;7(29):4615846172. doi:10.18632/oncotarget.10064

44. Bonnet M, Buc E, Sauvanet P, et al. Colonization of the human gut by E. coli and colorectal cancer risk. Clin Cancer Res. 2014;20 (4):859-867. doi:10.1158/1078-0432.CCR-13-1343

45. Yoon H, Kim N, Park JH, et al. Comparisons of gut microbiota among healthy control, patients with conventional adenoma, sessile serrated adenoma, and colorectal cancer. J Cancer Prev. 2017;22 (2):108-114. doi:10.15430/JCP.2017.22.2.108

46. Kinross J, Mirnezami R, Alexander J, et al. A prospective analysis of mucosal microbiome-metabonome interactions in colorectal cancer using a combined MAS 1HNMR and metataxonomic strategy. Sci Rep. 2017;7(1):8979. doi:10.1038/s41598-017-08150-3

47. Cremonesi E, Governa V, Garzon JFG, et al. Gut microbiota modulate T cell trafficking into human colorectal cancer. Gut. 2018;67 (11):1984-1994. doi:10.1136/gutjnl-2016-313498

48. Yan X, Liu L, Li H, Qin H, Sun Z. Clinical significance of Fusobacterium nucleatum, epithelial-mesenchymal transition, and cancer stem cell markers in stage III/IV colorectal cancer patients. Onco Targets Ther. 2017;10:5031-5046. doi:10.2147/OTT.S145949

49. Lennard KS, Goosen RW, Blackburn JM. Bacterially-associated transcriptional remodeling in a distinct genomic subtype of colorectal cancer provides a plausible molecular basis for disease development. PLoS One. 2016;11(11):e0166282. doi:10.1371/journal.pone.016 6282

50. Phipps AI, Limburg PJ, Baron JA, et al. Association between molecular subtypes of colorectal cancer and patient survival. Gastroenterology. 2015;148(1):77-87 e72. doi:10.1053/j.gastro. 2014.09.038

51. Seppala TT, Bohm JP, Friman M, et al. Combination of microsatellite instability and BRAF mutation status for subtyping colorectal cancer. Br J Cancer. 2015;112(12):1966-1975. doi:10.1038/bjc.2015.160

52. Manceau G, Marisa L, Boige V, et al. PIK3CA mutations predict recurrence in localized microsatellite stable colon cancer. Cancer Med. 2015;4(3):371-382. doi:10.1002/cam4.370

53. Flanagan L, Schmid J, Ebert M, et al. Fusobacterium nucleatum associates with stages of colorectal neoplasia development, colorectal cancer and disease outcome. Eur J Clin Microbiol Infect Dis. 2014;33 (8):1381-1390. doi:10.1007/s10096-014-2081-3 


\section{Publish your work in this journal}

Cancer Management and Research is an international, peer-reviewed The manuscript management system is completely online and includes open access journal focusing on cancer research and the optimal use of preventative and integrated treatment interventions to achieve improved outcomes, enhanced survival and quality of life for the cancer patient.

a very quick and fair peer-review system, which is all easy to use. Visit http://www.dovepress.com/testimonials.php to read real quotes from published authors.

Submit your manuscript here: https://www.dovepress.com/cancer-management-and-research-journal 